\title{
Les défis de l'orientation dans le monde
}

Introduction

\section{Georges Solaux}

\section{CpenEdition}

\section{Journals}

Édition électronique

URL : http://journals.openedition.org/ries/1415

DOI : $10.4000 /$ ries. 1415

ISSN : 2261-4265

Éditeur

Centre international d'études pédagogiques

Édition imprimée

Date de publication : 1 avril 2005

Pagination : 19-24

ISSN : 1254-4590

Référence électronique

Georges Solaux, "Les défis de l'orientation dans le monde », Revue internationale d'éducation de Sèvres [En ligne], 38 | avril 2005, mis en ligne le 18 novembre 2011, consulté le 22 septembre 2020. URL http://journals.openedition.org/ries/1415; DOI : https://doi.org/10.4000/ries.1415

(c) Tous droits réservés 


\title{
Les défis de l'orientation dans le monde
}

\section{Introduction}

\author{
Georges Solaux
}

La décision de réaliser un numéro spécial sur l'orientation scolaire dans le monde pourrait laisser croire que la totalité des articles présentés s’inscrit dans un cadre d'ensemble logique répondant à l'une ou a plusieurs des méthodes des comparaisons internationales rappelées par Michel Lallement ${ }^{1}:$ « rendre raison d'un fait social considéré comme universel et montrer dans quelle mesure chaque culture ne fait qu'accommoder... spécifier les propriétés uniques d'une société... mettre en évidence l'universalité d'un phénomène... spécifier les formes d'articulation spatio-temporelle d'un phénomène... ». Tel n'est pas le cas et la production d'articles homogènes construits selon un format strictement défini au préalable n'a pas été voulue car l'objet «orientation » est complexe et il peut être étudié selon des points de vue très différents. Le caractère essentiel de ce numéro se situe donc dans la variété des points de vue dont l'origine peut être trouvée dans les points qui suivent.

Dans un avis récent (Avis $\mathrm{n}^{\circ} 12$ - mars 2004), le Haut Comité d'évaluation de l'école (HCéé, France) précise par exemple que « le pilotage de la politique d'orientation consiste à gérer la contradiction entre deux objectifs qui s'entrecroisent en permanence : celui de la "gestion des flux" qui vise à répartir les élèves entre les différentes formations existantes et celui du "projet individuel” qui veut donner à un jeune les moyens d'effectuer progressivement ses choix de formation et de carrière en fonction de ses compétences et de ses souhaits ». Les enjeux de l'orientation des élèves sont ainsi clairement situés au niveau des équilibres à installer entre liberté individuelle et contrainte collective (voir l'article sur la France).

\section{UNE DIVERSITÉ DE POINTS DE VUE SUR UNE DIVERSITÉ DE SITUATIONS}

L'objet «orientation » peut en effet être indifféremment appréhendé selon une perspective macroscopique (les relations qu'entretient tel système d'orientation avec l'organisation administrative et la législation du pays, les

1. Lallement, M. (2004) «Apports et enjeux de la comparaison internationale », Sciences humaines, hors-série $\mathrm{n}^{\circ} 46$. 
résultats de l'orientation et l'adéquation formation - emploi, l'analyse des flux scolaires...), mais aussi dans une perspective microscopique centrée sur les individus en situation de choix (approche des psychologues et mode d'approche de certaines écoles de sociologie...). La diversité disciplinaire des auteurs s'inscrit dans cette volonté d'illustrer la variété des approches possibles. Certains auteurs sont économistes (Chevaillier, Paul), d'autres sont sociologues (Boulahcen, Solaux, Chiang), psychologues (Demeuse, Moumoula, Bakyono/ Nabaloum), juriste et politologue (Legrand).

Le souci de présenter l'orientation scolaire dans des contextes nationaux variés constitue une seconde source de différenciation des approches. Il a en effet été décidé de ne pas limiter l'approche aux pays riches ni aux pays francophones. Pour cette raison, nous avons sollicité l'intervention de collègues issus de pays riches à développement humain élevé (Belgique, Angleterre, France, Taïwan), de pays à développement humain moyen (Brésil, Laos, Maroc), de pays en développement à développement humain faible (BurkinaFaso). Dans certains pays, des réformes récentes nécessitent de centrer le texte sur la nouveauté (réforme des services d'orientation en Angleterre, l'accès dans le supérieur au Brésil et à Taïwan...) au détriment d'analyses sur la longue durée. Dans d'autres pays où les choses semblent plus stables sur le plan de l'organisation, la dimension diachronique prend le dessus. Enfin, les problèmes relatifs à l'orientation ne se situent pas au même niveau selon les pays : si Taïwan tente d'organiser l'admission dans le supérieur, le Maroc est davantage préoccupé par l'orientation à l'issue du collège et le Laos par la progression des taux de scolarisation dans le primaire.

Les données qui suivent sont issues de la publication $2004 \mathrm{du}$ Programme des Nations Unies pour le Développement (PNUD). Elles illustrent la très grande diversité économique et sociale de ces pays ${ }^{2}$ :

\footnotetext{
2. Alphabétisation des adultes : pourcentage des personnes âgées de quinze ans et plus qui peuvent, en le comprenant, lire et écrire un texte simple et court sur leur vie quotidienne.

Espérance de vie à la naissance : nombre d'années que vivrait un nouveau-né si les caractéristiques de mortalité de sa population au moment de sa naissance demeuraient les mêmes tout au long de sa vie.

PIB (produit intérieur brut) : somme de la valeur ajoutée par tous les producteurs résidant dans une économie, majorée de toutes les taxes sur les produits (hors subventions) non incluses dans la valorisation de la production. Le calcul du PIB ne tient pas compte des déductions au titre de l'amortissement des biens d'équipement manufacturés ou de l'épuisement et de la dégradation des ressources naturelles. La valeur ajoutée correspond à la production nette d'un secteur, après addition de toutes les composantes de cette production et déduction des facteurs intermédiaires.

Parité de pouvoir d'achat (PPA) : taux de change destiné à neutraliser les différences de prix entre pays, afin de permettre les comparaisons internationales de la production et du revenu en termes réels. Avec un dollar exprimé en parité de pouvoir d'achat dans un pays donné (comme c'est toujours le cas dans le présent rapport), il est possible de se procurer la même quantité de biens et de services que s'il s'agissait d'un dollar des États-Unis dans le cadre de l'économie de ce pays, ainsi que pour l'utilisation, par le biais d'accords

Scolarisation, taux brut de scolarisation combiné pour les écoles d'enseignement primaire, secondaire et supérieur : élèves inscrits dans un cycle d'enseignement primaire, secondaire et supérieur, quel que soit leur âge, exprimé en pourcentage de la population totale d'individus appartenant au groupe d'âge correspondant pour les trois niveaux.
} 


\begin{tabular}{|c|c|c|c|c|c|}
\hline Pays & $\begin{array}{c}\text { Espérance } \\
\text { de vie }\end{array}$ & $\begin{array}{c}\text { Alphabétisation } \\
\text { des adultes }\end{array}$ & $\begin{array}{c}\text { Taux de } \\
\text { scolarisation } \\
\text { combiné } \\
\text { de } 6 \text { à } 22 \text { ans }\end{array}$ & $\begin{array}{c}\text { PIB par tête } \\
\text { en PPA }\end{array}$ & $\begin{array}{c}\% \text { de } \\
\text { population } \\
\text { de moins } \\
\text { de } 15 \text { ans } \\
\text { sur pop. totale }\end{array}$ \\
\hline Taïwan $^{3}$ & 75,8 & 96 & 93,8 & 12916 & 19,8 \\
\hline Belgique & 78,7 & 100 & 111 & 27500 & 17,2 \\
\hline Royaume-Uni & 78,1 & 100 & 113 & 26150 & 18,7 \\
\hline France & 78,9 & 100 & 91 & 26920 & 18,6 \\
\hline Brésil & 68 & 86,4 & 92 & 7770 & 28,3 \\
\hline Laos & 54,3 & 66,4 & 59 & 1720 & 27,9 \\
\hline Maroc & 68,5 & 50,7 & 57 & 3810 & 36,8 \\
\hline Burkina-Faso & 45,8 & 12,8 & 22 & 1100 & 48,9 \\
\hline $\begin{array}{l}\text { Pays } \\
\text { développement }\end{array}$ & 64,6 & 76,7 & 60 & 4054 & 32,2 \\
\hline OCDE & 77,1 & & 87 & 24900 & 20,2 \\
\hline Monde & 66,9 & & 64 & 7804 & 29,4 \\
\hline
\end{tabular}

La lecture du tableau montre les différences nationales: entre la Belgique qui dispose d'un PIB/tête exprimé en PPA de 27500 dollars, d'une population de six à vingt-deux ans entièrement scolarisée et d'un poids de jeunes à prendre en charge relativement faible représentant 17,2\% de la population totale, et le Burkina-Faso, dont le PIB/tête en PPA est limité à 1100 dollars, dont un cinquième de la tranche d'âge est scolarisée, avec une prise en charge de jeunes de moins de quinze ans représentant pratiquement la moitié de la population totale, il ne semble pas possible d'imaginer une homogénéisation des discours sur les pratiques de sélection scolaire.

Ces chiffres traduisent également des différences sur le plan de l'organisation économique (ici une économie informelle, là une économie industrielle ou de service parfaitement intégrée dans la mondialisation), sur le plan des ressources financières (ici des pays bailleurs de fonds, là des pays surendettés), sur le plan de l'organisation administrative (ici des pays plutôt centralisés comme le Laos, là des organisations plus libérales comme en Belgique ou l'Angleterre, ou encore une diversité institutionnelle comme au Brésil), sur le plan de l'autonomie (aspect plus ou moins récent de la conquête des indépendances).

La variété de la richesse des pays s'accompagne de capacités différentes de financement de l'éducation :

3. Données non disponibles au PNUD, et relevant de diverses sources d'information. 


\begin{tabular}{|l|c|c|}
\hline \multicolumn{1}{|c|}{ Pays } & $\begin{array}{c}\text { Dépenses publiques } \\
\text { en \% du PIB }\end{array}$ & $\begin{array}{c}\text { \% des dépenses publiques } \\
\text { affectées au primaire }\end{array}$ \\
\hline Belgique & 5,8 & 33,3 \\
\hline Royaume uni & 4,6 & 34,4 \\
\hline France & 5,7 & 31,2 \\
\hline Brésil & 4 & 38,7 \\
\hline Laos & 3,2 & 46,9 \\
\hline Maroc & 5,1 & 48 \\
\hline Burkina-Faso & 2,7 & 45,7 \\
\hline
\end{tabular}

\section{AU-DELÀ dE CETTE DIVERSITÉ DES APPROCHES COMMUNES}

L'ensemble des textes traite des résultats de l'orientation en se fondant sur les notions communes d'efficacité et d'équité. La mobilisation des ressources affectées à la scolarisation peut être relativement efficace en matière de résultats produits par le système et/ou relativement équitables en matière d'équité dans l'allocation des ressources publiques aux différents groupes sociaux. Les articles présentés font peu ou prou référence aux résultats de l'orientation des élèves généralement analysés en termes d'efficacité et d'équité. On distingue généralement l'efficacité interne de l'efficacité externe. Le terme d'efficacité interne concerne ici les flux des élèves: taux de promotion, de redoublements, d'abandons, résultats aux examens. (voir les articles sur le Laos et le Burkina). Le calcul de l'efficacité interne résulte du rapport des ressources qu'utilise actuellement le système pour produire ses diplômés et des ressources que le système nécessiterait si le même nombre de diplômés était produit sans abandons ni redoublements. L'efficacité externe relève de la mesure des d'effets de l'éducation hors du système éducatif notamment au niveau de l'accès à l'emploi (voir article sur l'Angleterre), des carrières des salariés, de la durée du chômage, des salaires...

L'équité par l'accès à l'éducation : les calculs d'équité permettent quant à eux de vérifier que les enfants ont des chances de scolarisation aussi égales que possible. Les facteurs de variation traditionnellement utilisés pour réaliser cette vérification concernent les différences géographiques (entre provinces, entre le milieu rural et le milieu urbain, se référer aux articles sur le Burkina Faso et le Laos), les différences sexuées (les filles et les garçons, voir l'article sur le Laos), les différences socioéconomiques (différences selon les catégories sociales lorsqu'il est possible de disposer de l'information : voir l'article sur le Maroc)...

Les textes font également apparaître les outils de l'orientation : existence de services publics ou privés de conseil ou d'orientation, pertinence et mise à disposition des informations disponibles sur l'évolution des formations, 
des emplois et des qualifications. Le Burkina Faso, le Maroc et le tout jeune service anglais d'orientation en sont des exemples.

D’une manière générale, les mécanismes institutionnels de l'orientation scolaire retenus par les différents pays s'inscrivent dans le cadre général de l'organisation politique et administrative de chacun d'eux. Il s'agit en réalité d'un problème de régulation, d'arbitrage entre intérêts privés (la place laissée à l'initiative individuelle en matière de choix d'options ou de parcours scolaires) et d'intérêts publics (les besoins de la planification économique, la régulation de l'équité d'accès). La gestion formelle des principes de liberté et d'égalité est au cœur des mécanismes d'orientation. Les différents articles situent les procédures existantes dans les pays retenus entre ces deux pôles, sans que le choix préférentiel de l'un s'accompagne obligatoirement de l'abandon de l'autre (voir les différences entre le Maroc, la Belgique, le Brésil, la France).

\section{ORGANISATION DE LA PRÉSENTATION DES TEXTES}

Nous proposons de regrouper les huit textes en trois ensembles. L'objet traité dans chaque ensemble est particulier : l'orientation au collège pour le premier, l'admission et l'orientation dans l'enseignement supérieur pour le second, les politiques d'orientation pour le troisième. Chaque ensemble est en outre organisé de telle sorte que la diversité économique, politique et scolaire initiale soit peu ou prou respectée au sein de chacun d'eux.

La problématique de l'orientation en Belgique et au Maroc est traitée par le biais de l'orientation au collège et de la place centrale que ce dernier occupe dans l'orientation-répartition-tri des élèves avant la fin de leur scolarité obligatoire dans les filières générales, ou technologiques, ou professionnelles, ou dans la vie active. Si, au Maroc, "la Carte scolaire, concrétisant la volonté socio- politique de l'État, fixe le taux d'orientation à $47 \%$ en moyenne de l'ensemble des élèves de la $3^{\circ}$ ASC (quel que soit l'établissement scolaire - et donc que ce soit un collège à population plus bourgeoise, plus populaire ou typiquement rurale - la même règle s'applique de façon stricte concernant le passage) " 4 , en Belgique c'est "l'établissement scolaire et, plus particulièrement, le conseil de classe, qui exerce la fonction d'orientation et de sélection ». Le terme " orientation ", dans l'enseignement secondaire, est bien souvent associé à "attestation d'orientation ». Ce terme renvoie à l'avis émis par le conseil de classe à l'issue de chaque année scolaire et qui prend, pour chaque élève, l'une des trois formes suivantes : "l'élève est admis à poursuivre " (attestation d'orientation A ou AOA), "l'élève est admis à poursuivre avec des restrictions qui limitent le choix des filières et options » $(\mathrm{AOB})$ ou « l'élève doit redoubler son année »(AOC).

4. Toutes les citations qui suivent sont extraites des articles. 
Les articles consacrés à Taïwan, au Brésil et dans une moindre mesure au Burkina Faso, analysent l'orientation à l'issue de l'enseignement secondaire. Si les données produites sur le Burkina Faso concernent l'ensemble des niveaux scolaires, il nous paraît que le problème de l'admission dans l'enseignement supérieur en pays en développement est tellement bien posé que nous le regroupons avec les deux précédents : alors que le baccalauréat permettait l'admission directe à l'université, l'enseignement supérieur n'est plus « considéré comme un aboutissement légal et naturel de l'enseignement secondaire. Sa finalité doit être de former les élites ». Le texte sur Taïwan " expose les caractéristiques, arguments et problèmes » de deux modalités de sélection pour l'entrée à l'université : «l'examen commun d'entrée à l'université, ainsi que le programme qui lui a succédé, le programme d'admission diversifiée pour les universités ». Enfin, l'admission dans l'enseignement supérieur et l'orientation des étudiants au sein et à l'issue de celui-ci sont analysés pour le Brésil en inscrivant clairement les données dans le cadre institutionnel particulier du pays. "L'enseignement supérieur brésilien est constitué d'établissements publics (municipaux, d'État ou fédéraux) et privés, qui peuvent être soit des universités (c'est le cas de la plupart des institutions publiques), soit des facultés centrées sur un nombre limité de disciplines. Ce qui pourra étonner un Européen, c'est la domination numérique exercée par l'enseignement privé, qui accueille $70 \%$ des étudiants, et qui représente presqu'une institution sur dix ».

Le troisième ensemble d'articles est centré sur l'inscription des politiques d'orientation dans le cadre plus général des politiques publiques. Il en est ainsi du texte sur le Laos qui montre comment l'organisation relativement centralisée de l'État s'accompagne d'une orientation régulée à tous les niveaux par les examens. Les récentes réformes des services d'orientation anglais sont emblématiques de la libéralisation du début des années quatre-vingts et des recentrages réalisés ces dernières années : « de 1991 à 1995, en Angleterre, en Écosse et au Pays de Galles, le réseau du service public d'orientation a été privatisé ». Un nouveau service, appelé Connexions, fut créé en 2001 " réservé aux jeunes de treize à dixneuf ans (les “teen-agers"), il a été conçu comme un grand "service de la jeunesse" et ne se limite pas à l'orientation mais propose tout un ensemble de services et de conseils (aides financières, logement, santé, drogue, contraception, etc.)...». Enfin, il semble bien que l'évolution des décisions des gouvernements successifs à l'égard des services d'orientation français traduisent les hésitations des partis politiques quant aux mesures à prendre pour installer l'organisation administrative dans une perspective libérale : "projection dans l'espace scolaire d'enjeux extérieurs à l'École... l'orientation recouvre une politique de contrôle et de planification des flux d'élèves qui utilise de façon privilégiée la réforme des structures...» ou alors elle "vise à faciliter la construction du projet personnel; elle s'attache davantage aux individus... ». 